\title{
Atypical organisation of the auditory cortex in dyslexia as revealed by MEG
}

\author{
Sabine Heim ${ }^{\mathrm{a}, *}$, Carsten Eulitz ${ }^{\mathrm{a}}$, Jürgen Kaufmann ${ }^{\text {a }}$, Ivonne Füchter ${ }^{\mathrm{a}}$, \\ Christo Pantev ${ }^{b}$, Antoinette Lamprecht-Dinnesen ${ }^{c}$, Peter Matulat ${ }^{c}$, Petra Scheer ${ }^{c}$, \\ Marianne Borstel ${ }^{\mathrm{c}}$, Thomas Elbert ${ }^{\mathrm{a}}$ \\ a Department of Psychology, University of Konstanz, P.O. Box D25, 78457 Konstanz, Germany \\ ${ }^{\mathrm{b}}$ Institute of Experimental Audiology, University of Münster, Münster, Germany \\ ${ }^{\mathrm{c}}$ Department of Phoniatrics and Pedaudiology, University of Münster, Münster, Germany
}

Received 4 August 1999; received in revised form 10 March 2000; accepted 17 May 2000

\begin{abstract}
Neuroanatomical and -radiological studies have converged to suggest an atypical organisation in the temporal bank of the left-hemispheric Sylvian fissure for dyslexia. Against the background of this finding, we applied high temporal resolution magnetoencephalography (MEG) to investigate functional aspects of the left-hemispheric auditory cortex in 11 right-handed dyslexic children (aged 8-13 years) and nine matched normal subjects (aged 8-14 years). Event-related field components during a passive oddball paradigm with pure tones and consonant-vowel syllables were evaluated. The first major peak of the auditory evoked response, the M80, showed identical topographical distributions in both groups. In contrast, the generating brain structures of the later M210 component were located more anterior to the earlier response in children with dyslexia only. Control children exhibited the expected activation of more posterior source locations of the component that appeared later in the processing stream. Since the group difference in the relative location of the M210 source seemed to be independent of stimulus category, it is concluded that dyslexics and normally literate children differ as to the organisation of their left-hemispheric auditory cortex.
\end{abstract}

Keywords: Dyslexia; Magnetoencephalography (MEG); Auditory evoked magnetic field; Children

\section{Introduction}

The term dyslexia refers to children who have difficulties in learning to read, write and spell, despite evidence of sensory integrity, normal intellectual capacity, as well as adequate educational and sociocultural opportunities [2]. Prevalence estimates varying between 4 and $9 \%$ [42] point to the epidemiological validity of the disorder. Although the biological basis is still under debate, dyslexia is believed to reflect deficits in the functioning of the central nervous system [36].

Given the complexity of cognitive processes involved in reading and writing, it is not surprising that a wide range of possible etiological models have been offered for

\footnotetext{
* Corresponding author. Tel.: + 49-7531-883301; fax: + 49-7531882891.

E-mail address: sabine.heim@uni-konstanz.de (S. Heim).
}

dyslexia. A plethora of research exists supporting three models that associate dyslexia with: (1) low-level visual processing difficulties due to a selective impairment in the magnocellular layers of the lateral geniculate nucleus (for a review see Ref. [10]); (2) a general auditory processing deficit, specifically in temporal coding of rapid auditory changes as, for instance, in transition periods of consonant-vowel syllables (for reviews see Refs. [7,48]); and (3) speech sound processing problems arising from poor phonological awareness (for reviews see Refs. [37,52]).

Anatomical and radiological studies of dyslexic brains suggest striking alterations in the temporal bank of the left-hemispheric Sylvian fissure [9,45]. The region within and surrounding the Sylvian fissure contains structures playing a specialized role in the analysis of acoustic signals. One prominent structure is the planum temporale, a plane caudal to the first Heschl's gyrus [51]. Because the left-hemispheric planum builds a part of 
the Wernicke's speech comprehension area and because it is, on average, larger in size in normal right-handed adults than the corresponding structure of the right hemisphere (for a review see Ref. [46]), the planum temporale has often been considered as an important biological substrate of language lateralization.

The impetus of focusing attention on the planum temporale in dyslexic children has been given from post-mortem studies of Galaburda et al. (for reviews see Refs. [8,9]). A number of post-mortem and in vivo studies have revealed either a reduced or an absence of gross left-right asymmetry of the planum temporale in people with dyslexia [11,15,16,19,24]. A magnetic resonance imaging (MRI) study by Larsen et al. [20] suggests that right-handed dyslexic subjects with phonological processing deficits might display symmetrical plana. Galaburda et al. [12] presented evidence that the symmetry is due to a larger right rather than a smaller left planum. Generalizing this finding Galaburda et al. proposed that symmetrical brains of dyslexics result from a diminished neural loss in the right hemisphere during prenatal development. It deserves mention, however, that more recent MRI techniques have failed to find significant changes in normal planum temporale asymmetry in people with dyslexia [21,39,41]. Variations in subject characteristics (i.e. criteria used to define dyslexia, heterogeneity of the dyslexic population) and differences in anatomical definition of the planum temporale may play an important role in explaining these conflicting results.

During the performance of phonological tasks, Shaywitz et al. [43] observed a relatively reduced blood flow in Wernicke's area, angular gyrus and striate cortex and a relative overactivation in frontal brain regions (e.g. Broca's speech area) of dyslexic as compared to normal adults, by means of functional magnetic resonance imaging (fMRI). A similar left-hemispheric activation pattern was obtained with positron emission tomography (PET) by Brunswick et al. [1] during an explicit reading experiment. Relative to normal controls, dyslexic readers showed reduced activation in the left posterior inferior temporal cortex (Wernicke's 'Wortschatz' or thesaurus), left cerebellum, left thalamus and medial extrastriate cortex and enhanced activation in a pre-motor region of Broca's area. When reading was implicit, dyslexic subjects also demonstrated diminished activation in Wernicke's Wortschatz, but no elevated activation in anterior parts of the brain.

Auditory ERP components, like the P50 and N100, have provided another tool to evaluate information processing in children with different handicaps $[31,44,49]$. From a developmental standpoint, the P50 and N100 appear to be robust components, although reports about the peak latencies in children vary extremely (e.g. $[17,26,32,47,50])$.

The ERP component 'mismatch negativity' (MMN) is elicited by deviant stimuli (e.g. minimal changes in frequency, intensity or duration) occurring within a block of repetitive homogenous standard stimuli (for a review see Ref. [30]). The response is believed to reflect a neuronal mismatch between the deviant auditory input and a sensory memory trace storing the physical features of the standard stimulus. Kraus et al. [18] report a nearly absent mismatch response to deviant consonant-vowel syllables in learning impaired children when compared to normal controls. Moreover, this result was correlated with behavioural discrimination of this rapid changing speech stimuli (/da/ versus /ga/) leading Kraus et al. to suggest that the phonemic discrimination deficits likely have their origins in the auditory pathways and are pre-attentive in nature. Schulte-Körne et al. [40] observed that the MMN to speech sounds (/ba/ versus /da/) in dyslexic boys was attenuated but not absent.

Particular attention has been paid to the source locations of the P50, N100 and MMN, especially by means of magnetoencephalography (MEG). MEG studies have demonstrated that corresponding auditory event-related fields (ERFs) - P50m, N100m and 'mismatch field' (MMF) - have sources in the supratemporal auditory cortex (for a review see Refs. [14,35,56]). However, it is important to distinguish between the structures generating the event-related components and the underlying causes for a deviance in these responses. Sources in the supratemporal cortex, for instance, can be influenced by bottom-up processing in the auditory pathway or top-down processing occurring in other parts of the brain.

Taken together, high temporal resolution electrophysiological procedures are particularly able both to detect atypical information processing in children and to reveal general functional principles of the brain. In particular, the MEG is a very appropriate tool to study auditory processing and functional organisation in developmental disorders like dyslexia for the following reasons: (1) Neuromagnetic fields are generated by intracellular currents in pyramidal neurones of fissural cortex flowing tangentially to the scalp. Hence, the supratemporal auditory cortex, located as it is in the Sylvian fissure, is well-positioned for MEG recordings. (2) The high temporal resolution of MEG may be capable of reflecting central auditory processing with an accuracy of the order of milliseconds. Thus, MEG is a good technique to address ideas about auditory processing deficits in dyslexia, as suggested by Tallal [48]. (3) MEG recordings are non-invasive and free of bodily contact, i.e. require no electrode placement on the scalp as required for the EEG. (4) At instances in time when there is only one focal source of activation per hemisphere, magnetic source imaging allows the determination of the source location with an accuracy of a few millimetres [5,25].

The purpose of the present study was to investigate functional aspects of the left-hemispheric auditory cortex response to speech sounds in dyslexic children by 
Table 1

Psychological assessment data across groups ${ }^{\mathrm{a}}$

\begin{tabular}{llccc}
\hline & & Controls $(n=9)$ & Dyslexics $(n=10)$ & $U^{\mathrm{d}}$ \\
\hline $\begin{array}{l}\text { Non-verbal intelligence } \\
\text { Written language scores }\end{array}$ & $\mathrm{IQ}^{\mathrm{c}}$ & 120 & 111.5 & 27.5 \\
Standard reading & \% Rank (bandwidth) & $50-100$ & $0-25$ & 0.1531 n.s. \\
Pseudoword reading & Errors $^{\mathrm{c}}$ (max. $\left.=30\right)$ & 1 & 12 & 8.5 \\
Standard spelling & \% Rank & 81 & 13 & 0.0029 \\
\hline
\end{tabular}

\footnotetext{
${ }^{a}$ For further illustration see text. n.s., non-significant.

${ }^{\mathrm{b}}$ Mode.

${ }^{\mathrm{c}}$ Median.

${ }^{\mathrm{d}}$ Mann-Whitney $U$-test.
}

utilizing the benefits of MEG and magnetic source imaging. In the course of this, the first two major ERF components M80, M210 and in addition, the MMF were evaluated. Based on anatomical and physiological findings, we put forward two hypotheses: (1) There is a difference in the organisation of the left auditory cortex - particularly revealed by differences in source locations of M80 and M210 components - between dyslexics and normal controls. (2) Dyslexics display an absent MMF to deviant consonant-vowel syllables that might reflect a psychoacoustic dysfunction in a time frame of some tens of milliseconds.

\section{Methods}

\subsection{Subjects}

Eleven dyslexic children (two girls) were identified from the records of the outpatient clinic at the Department of Phoniatrics and Pedaudiology of the Münster University. Diagnosis of dyslexia was based on a discrepancy ( $\geq 1$ S.D.) between actual spelling scores and spelling predicted by general mental ability [40]; dyslexic subjects were also required to show poorer than normal reading achievement. Nine normal controls were matched for age, sex (one girl), mother tongue (all native speakers of German) and handedness (all right-handed). One dyslexic boy was excluded from further analyses because of insufficient data quality (movement artefacts during recording). Mean age was 10.50 years (range: $8-13$ years; S.D. $=1.72$ ) and 10.78 years (range: $8-14$ years; S.D. $=2.28$ ) for dyslexic and control groups, respectively.

Subjects had no history of neurological, otological or psychiatric disease. Furthermore, none of the children were on medication that might affect the central nervous functioning. As shown in Table 1, written language skills were documented by pseudoword reading ${ }^{1}$ [55], standard

\footnotetext{
${ }^{1}$ Normally the administered test demands verbal repetition of spoken pseudowords, two to six syllables in length.
}

reading (Zürcher Lesetest [ZLT] [23]) and spelling tests (Diagnostischer Rechtschreibtest [DRT] for Grade 2, 3, 4 or 5 [13,27-29]; Westermann Rechtschreibtest for Grades 6-8 [WRT 6+] [38]). Mental ability was assessed by age-appropriate German adaptations of Cattell's Culture Fair Intelligence Test, CFT 1 or CFT 20 $[53,54]$. The CFT provides a non-verbal measure on intelligence, thus reducing the influence of verbal abilities and education on IQ test performance.

The nature of the study was fully explained to the subjects' parents and their consent obtained. Every child received a cinema ticket and a surprise trinket for her/his voluntary participation.

\subsection{Magnetoencephalographic measures}

\subsubsection{Stimulation}

Three different blocks of stimuli were presented through ear tubes (see Ref. [35]) to the subject's right ear (contralateral to the MEG-investigated hemisphere ${ }^{2}$ ) with a constant intertrial interval (stimulus onset to stimulus onset) of $1 \mathrm{~s}$ and a stimulus duration of $200 \mathrm{~ms}$. Blocks 1 and 2 included 1000 stimuli each of natural spoken syllables $/ \mathrm{da} /$ and $/ \mathrm{ga} /$, while 500 sine wave tones ${ }^{3}$

Table 2

The five difference $(\Delta)$ wave forms ${ }^{\mathrm{a}}$

\begin{tabular}{ll}
\hline No. & Difference wave forms \\
\hline la. & $\Delta(/ \mathrm{ga} /-\mathrm{D}-/ \mathrm{da} /-\mathrm{S}) ;$ first occurring block \\
1b. & $\Delta(/ \mathrm{ga} /-\mathrm{D}-/ \mathrm{da} /-\mathrm{S}) ;$ second occurring block \\
2a. & $\Delta(/ \mathrm{da} /-\mathrm{D}-/ \mathrm{ga} / \mathrm{S}) ;$ first occurring block \\
2b. & $\Delta(/ \mathrm{da} /-\mathrm{D}-/ \mathrm{ga} /-\mathrm{S}) ;$ second occurring block \\
3. & $\Delta(1200 \mathrm{~Hz}-\mathrm{D}-1000 \mathrm{~Hz}-\mathrm{S})$
\end{tabular}

${ }^{\text {a }}$ For further illustration see text. D, deviant; S, standard.

\footnotetext{
${ }^{2}$ Previous studies have shown that the strongest auditory ERFs are recorded on the side contralateral to the side of handedness $[3,4]$ and stimulation $[3,4,34]$.

${ }^{3}$ The frequency-dependent representation of pure tones in primary auditory area should serve as a landmark (e.g. Ref. [35])
} 
(1000 and $1200 \mathrm{~Hz}$, with $10 \mathrm{~ms}$ rise and fall times) were presented in the third block (see also Table 2). The stimulus intensity was at $65 \mathrm{~dB}$ sensation level.

In each block, an oddball paradigm was employed in which a deviant stimulus $(\mathrm{D}$, probability of occurrence $10 \%$ ) was presented in a series of standards $(\mathrm{S}$, probability of occurrence $90 \%$ ): Block $1: / \mathrm{da} /=\mathrm{S}, / \mathrm{ga} /=\mathrm{D}$; Block 2: $/ \mathrm{ga} /=\mathrm{S}, / \mathrm{da} /=\mathrm{D}$; Block 3: $1000 \mathrm{~Hz}=\mathrm{S}, 1200$ $\mathrm{Hz}=\mathrm{D}$. Stimuli were presented in a pseudorandom order with at least six standard stimuli separating presentations of two deviants. To achieve a better signalto-noise ratio, the two blocks with verbal material were presented a second time, resulting in five blocks altogether. The order of the blocks was pseudorandomized, with no two identical blocks occurring consecutively.

To control the level of arousal as well as to constrain eye movements and blinks during recording, subjects watched silent cartoons or movies displayed on a special magnetic field free video screen. In addition, concentrating on the film helped the children to lie still during recording, which lasted $\approx 45 \mathrm{~min}$. The participants were instructed to attend to the video program and to ignore the auditory stimuli. They were also informed that the examiner would enter the recording room after every block and that slight movements of the lower body were permitted at this time. Two-way speakers and a video camera allowed the researchers to communicate with the child, who stayed alone in the magnetically shielded room during data acquisition.

\subsubsection{Data acquisition}

Recordings were performed inside a magnetically shielded room, using a 37-channel biomagnetometer (Magnes $^{\mathrm{TM}}$, Biomagnetic Technologies). The detection coils of the system are arranged in a circular concave array (diameter: $144 \mathrm{~mm}$ ) with a radius of $122 \mathrm{~mm}$. The axes of the detection coils are normal to the surface of the sensor array. The distance between the centres of two adjacent coils measures $22 \mathrm{~mm}$; each coil has a diameter of $20 \mathrm{~mm}$. The sensors are configured as first-order axial gradiometers with a baseline of $50 \mathrm{~mm}$. The spectral density of the intrinsic noise of each channel was between 5 and $7 \mathrm{fT} / \sqrt{ } \mathrm{Hz}$ in the frequency range $>1 \mathrm{~Hz}$. The children laid on their right side with their head, neck and upper body supported by a specially fabricated vacuum mattress. A sensor position indicator system determined the spatial locations of the sensors relative to the head and indicated if head movements occurred during recordings. The neuromagnetic field pattern was recorded over the left supratemporal cortex. The measurement system was centred over a point $\approx 1.5 \mathrm{~cm}$ superior to position $\mathrm{T} 3$ of the $10-20$ system for electrode placement (corresponds approximately to the midpoint of the Sylvian fissure) and was positioned as close as possible to the subject's head. Using a bandwidth of $0.01-100 \mathrm{~Hz}$ for filtering and a sampling rate of $297.6 \mathrm{~Hz}$, five blocks of 500 stimulusrelated epochs, each of $700 \mathrm{~ms}$ (including $100 \mathrm{~ms}$ prestimulus baseline), were recorded and stored for further analysis.

\subsubsection{Data analysis}

For every block of data, selective averages were computed from the responses to the standards and deviants separately for each stimulus class. Stimulus-related epochs that were contaminated by muscle or eye blink artefacts (signal variations of $>3.5 \mathrm{pT}$ ) were automatically rejected from the averaging procedure. A baseline correction was carried out for all channels by subtracting the mean value of the signal during the 100 $\mathrm{ms}$ prior to the stimulus. The data were low-pass filtered using a $20 \mathrm{~Hz}$ second-order zero-phase shift Butterworth filter (12 dB/oct).

In order to analyse the MMF, a difference wave was computed for each of the five blocks by subtracting the selective average responses to the standard stimulus from the selective average responses to the deviant stimulus (see Table 2). Due to the low strength of the five difference waves around $40 \mathrm{fT}$ in comparison to the biological noise, we decided not to assess the source locations of the mismatch generator. For comparisons of the mismatch waves, two evaluation intervals $(\mathrm{A}=$ $180-280 \mathrm{~ms}$ and $\mathrm{B}=280-380 \mathrm{~ms}$ ) were selected.

A single moving equivalent current dipole (ECD) model in a spherical volume conductor was used for source analyses. A sufficient data quality for this data analysis technique was achieved for standard stimuli. Therefore, source analysis was calculated for the M80 and M210 components in response to standard stimuli. Source parameters included location, dipole moment as well as parameters estimating the quality of the dipole fit, i.e. goodness of fit and confidence volume. Source locations were computed in a head-based Cartesian coordinate system. The origin of this coordinate system was set at the midpoint of the medial-lateral axis ( $y$-axis), the axis that joined the centre points of the entrance to the acoustic meatuses of the right and left ear (positive towards the left ear). The posterior-anterior axis ( $x$-axis) extended from the origin to the nasion (positive towards the nasion) and the inferiorsuperior axis ( $z$-axis) was perpendicular to the $x-y$ plane (positive towards the vertex). While the dipole moment $(q)$ reflects the strength of the located field (in $\mathrm{nAm}$ ), the goodness of fit value (in percent) describes how much of the measured magnetic field is accounted for by the ECD.

Analyses of the five standard response waves (/da/-S and /ga/-S first and second occurring block, $1000 \mathrm{~Hz}$ ) led to the estimated dipole locations of the M80 and M210. For each standard evoked magnetic field, a poststimulus time interval containing the field maximum and minimum (= evaluation interval in $\mathrm{ms}$ ), the 
local root mean square (RMS) maximum (in fT), and the local goodness of fit maximum were chosen. The spatial ECD coordinates $(x, y, z)$ for every component were assigned to the corresponding averaged values of five to eight adjacent sampling points $(16.75-26.80 \mathrm{~ms})$ around the centre of the obtained evaluation intervals. Only those $x, y$ and $z$ values were included in the average, which fulfilled the following additional criteria based on statistical and anatomical considerations: (a) goodness of fit $>90 \%$; (b) distance of ECD to midsagittal plane $>1.5 \mathrm{~cm}$; (c) inferior-superior value $>3$; and (d) confidence volume of $<0.1 \mathrm{~cm}^{3}$. Since the measured parameters ( $x, y, z, q$ and latency) of the M80 and M210 components in the two identical syllable blocks were very similar (test/retest criterion) the averaged data of these blocks were used for further analysis.

\subsection{Statistics}

The latencies, the RMS amplitudes, the dipole moment and the ECD locations (anterior-posterior, medial-lateral, inferior-superior) of the M80 and M210 were used as dependent variables in three-way analyses of variance (ANOVA). Group (dyslexics versus controls) was treated as between-group factor, component (M80 versus M210) and stimulus (/da/-S versus /ga/-S, averaged over two identical syllable blocks versus 1000 $\mathrm{Hz}$ ) as repeated measurement factors. Probability effects of more than two-level repeated measures factors were Greenhouse-Geisser adjusted. Post-hoc comparisons were carried out using the Scheffé test.

In the case of the MMF, a three-way ANOVA was computed with group treated as a between-group factor, interval (A versus B) and block (1-5, see also Table 2) as repeated measures. The dependent variable was the RMS amplitude.

\section{Results}

\subsection{M80 and M210}

\subsubsection{Latencies}

In response to the standard stimuli, a sequence of two prominent waves was easily detectable in all subjects. We labelled these deflections M80 and M210, as the latencies were distinctly longer than the corresponding set of deflections (P50m and N100m) in adults. ${ }^{4}$ An analysis of the latencies of the M80 (mean latency $=80$ $\mathrm{ms}$, range $=67 \mathrm{~ms}$ ) and $\mathrm{M} 210$ (mean latency $=211 \mathrm{~ms}$, range $=105 \mathrm{~ms})$ attained a significant main and interac-

\footnotetext{
${ }^{4}$ In his MEG study, Paetau et al. [33] have demonstrated major effects of interstimulus interval on the $\mathrm{N} 100 \mathrm{~m}$, with delayed or absent $\mathrm{N} 100 \mathrm{~m}$ in healthy children at rapid stimulation rates and adult type $\mathrm{N} 100 \mathrm{~m}$ responses at slower stimulation $(1.2-2.4 \mathrm{~s})$ rates.
}

tion effect for the factors component and stimulus. The component by stimulus interaction $[F(2,34)=5.6, P<$ $0.018, \varepsilon=0.685]$ as well as the main effect of the factor stimulus $[F(2,34)=10.7, \quad P<0.001, \varepsilon=0.777]$ result from shorter M210 latency to the standard tone (mean latency $=194 \mathrm{~ms})$ than to the standard syllables $/ \mathrm{da} /$ $($ mean latency $=221 \mathrm{~ms})$ and $/ \mathrm{ga} /($ mean latency $=219$ $\mathrm{ms}$ ), as indicated by significant post-hoc comparisons. The difference between the consonant-vowel syllables was insignificant. For the component M80 the tone$\mathrm{da}-$ ga contrasts (mean latency $=76,81$ and $84 \mathrm{~ms}$, respectively) did not reach significance. There were no significant group differences.

Fig. 1 demonstrates sets of magnetic field waveforms in response to the standard syllable /da/ for an individual dyslexic and control subject. Clear extrema and polarity reversals of the M80 (first deflection) and M210 (second deflection) become evident.

\subsubsection{Magnetic field amplitudes and dipole moments}

The three-way ANOVA of M80 and M210 RMS values obtained for the standard stimuli revealed significant main effects for the factors component $[F(1,17)=9.7, P<0.006]$ and stimulus $[F(2,34)=9.3$, $P<0.002, \varepsilon=0.798]$. The field amplitude of the M210 was larger than that of the M80 (mean RMS value = 101 vs. $71 \mathrm{fT}$, respectively). Finally, the two components exhibit greater signal power to syllables [mean RMS value (S.E.M.) for standard $/ \mathrm{da} /=88 \mathrm{fT}$ (5.47) and standard $/ \mathrm{ga} /=92 \mathrm{fT}(5.52)]$ than to the tone (mean $\mathrm{RMS}$ value $=78 \mathrm{fT}$, S.E.M. $=5.78$ ). The group factor yielded no statistically meaningful influence on field amplitudes. Possible interactions between the factors group, component and stimulus remained insignificant.

The results of the statistical analysis of RMS field amplitudes are confirmed by the corresponding dipole moment values. First, for both the dyslexics and controls the dipole moment of the M210 (mean $q$ value = $33 \mathrm{nAm}$ ) was nearly twice as large as that of the M80 (mean $q$ values $=18 \mathrm{nAm}$ ) elicited by standard stimuli $[F(1,13)=14.2, P<0.002]$. Second, the dipole moment is generally stronger in the syllable conditions (mean $q$ value for standard $/ \mathrm{da} /=28 \mathrm{nAm}$ and standard $/ \mathrm{ga} /=$ $30 \mathrm{nAm}$ ) than in the tone condition (mean $q$ value $=19$ nAm) $[F(2,26)=7.4, P<0.014, \varepsilon=0.648]$. Third, the group effect and possible interactions between the factors group, component and stimulus failed to attain significance.

\subsubsection{Source locations}

The $x, y$ and $z$ coordinates of the estimated source locations of the M80 and M210 obtained for the nonverbal and verbal standards are presented in Fig. 2.

Within each group, the two-dimensional plots indicate source clusters for the different M80 waves on the 
dyslexic subject

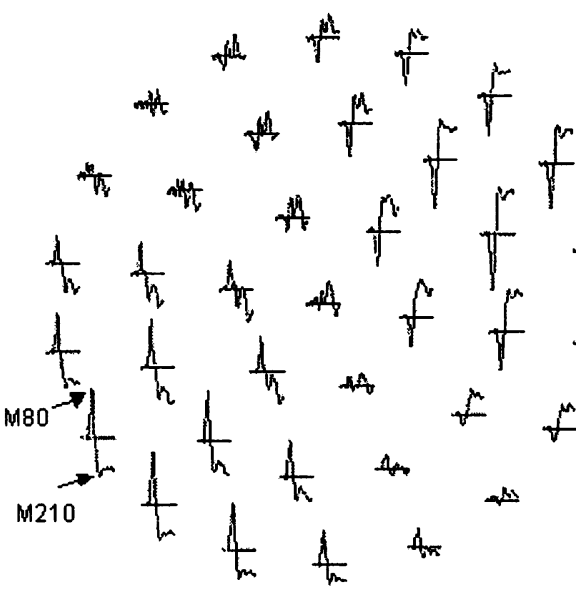

control subject

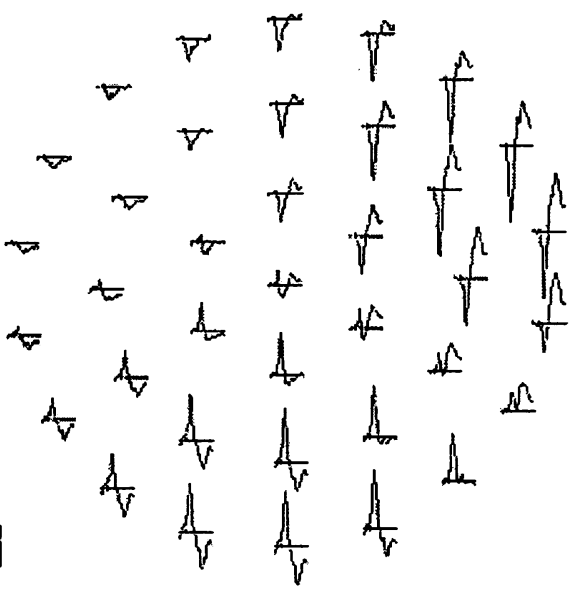

Fig. 1. Sets of 37 averaged wave forms in response to the standard syllable /da/ (second occurrence, see Table 2) measured over the left hemisphere in one dyslexic and one control subject. The centre of the sensor array corresponds to the point $\approx 1.5 \mathrm{~cm}$ superior to the position $\mathrm{T} 3$ (approximately the midpoint of the Sylvian fissure) of the 10-20 system for electrode placement of the subject. The responses are presented according to the placement of the pickup coils relative to the child's head. Flux leaving the skull is positive (outgoing magnetic field); flux entering the skull is negative (ingoing field). The first (positive) deflection represents the M80, the second (negative) deflection the M210. The epoch length is $700 \mathrm{~ms}$ and small vertical bars represent the stimulus onset.
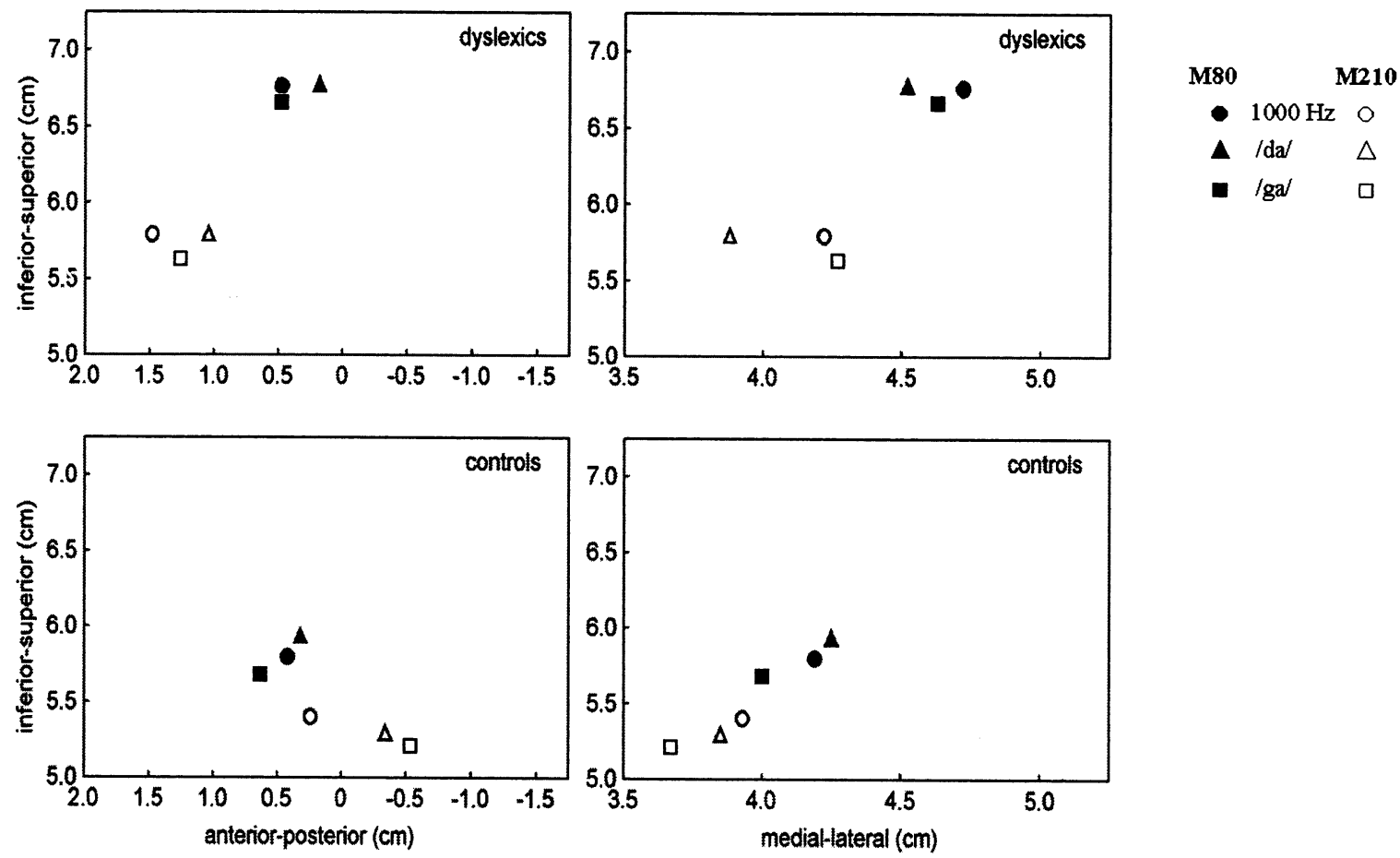

Fig. 2. Source locations of the M80 and M210 to the different standard stimuli for the dyslexic and the control group. The left 2D-plots represent anterior-posterior and inferior-superior axes, the right plots medial-lateral and inferior-superior axes.

one hand and M210 waves on the other, i.e. three-way ANOVAs of the $x, y$ and $z$ values yielded no significant Component $\times$ Stimulus interactions. Interestingly, the statistical analysis of the $x$ coordinate (posterior-ante- rior direction) revealed a highly significant group by component interaction $[F(1,13)=12.4, P<0.004]$. The overall significance is entirely due to the group contrast of the M210 localisation $(P<0.004)$ indicating that in 
Source locations of the M210
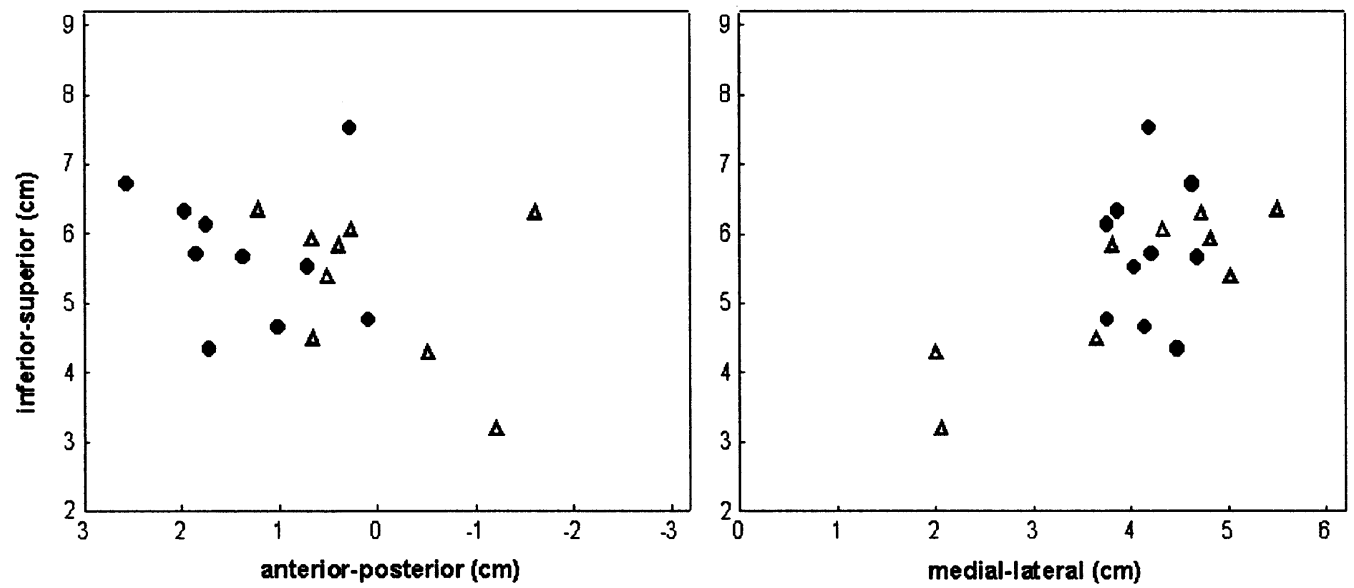

(a)

dyslexics

$\Delta$ controls

Localisations of the $\mathrm{MR10}$ relative to the source location of the M80
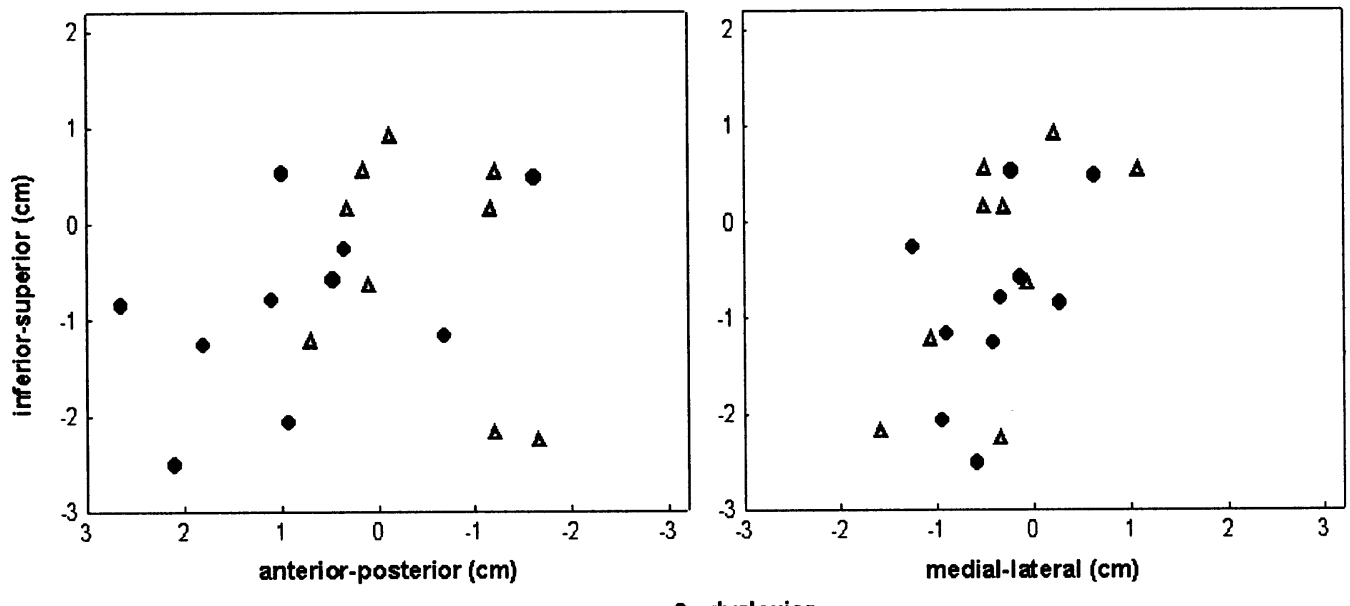

(b)

\section{dyslexics}

$\Delta$ controls

Fig. 3. Individual subject data from ten dyslexics and nine controls for source locations of the M210 averaged across tonal and verbal standard stimuli. The left 2D-plots represent anterior-posterior $(x)$ and inferior-superior axes, the right plots medial-lateral $(y)$ and inferior-superior axes (z). (A) Absolute locations of the M210 generator. (B) Localisations of the M210 relative to the source location of the M80. Mean coordinates (S.E.M.) are $x, y, z=[0.81,-0.39,-0.85(0.40,0.18,0.31)]$ and $x, y, z=[-0.46,-0.35,-0.43(0.28,0.25,0.39)]$ for dyslexic and control groups, respectively.

the dyslexics, the sources of the M210 are considerably more anterior than in the controls (mean $\mathrm{x}$ value $=1.26$ and $-0.21 \mathrm{~cm}$, respectively). This result is illustrated in Fig. 2 (left plots). For the $y$ and $z$ axis values, only the factor component had a pronounced impact $[F(1,14)=$ $6.8, P<0.021$ and $F(1,14)=8.0, P<0.013]$. As can be seen in Fig. 2 (right plots), the sources of the M210 are located more medial (mean $y$ value $=3.97 \mathrm{~cm}$ ) and more inferior (mean $z$ value $=5.52 \mathrm{~cm}$ ) than the corresponding equivalent sources of the M80 (mean $y$ value $=4.38 \mathrm{~cm}$, mean $z$ value $=6.27 \mathrm{~cm}$ )

Localisations of the M210 generator to tonal and verbal standard stimuli obtained from individual subjects in each group are displayed in Fig. 3A. To account for a possible effect of neuroanatomical variability (e.g. size of the head) on M210 source locations, localisations were normalized with respect to M80 as the difference $\Delta(\mathrm{M} 210-\mathrm{M} 80)$. These relative source locations are presented for each dyslexic and control subject in Fig. 3B.

Fig. 3B (left) underlines the group difference of the M210 locations in the anterior-posterior direction. The majority of the dyslexic subjects processed the standard tone and syllables at a more anterior place [mean group difference $\left.^{5}=1.27 \mathrm{~cm}, F(1,17)=6.3, P<0.023\right]$. On the other side, similar locations of acoustic processing of

\footnotetext{
${ }^{5}$ Mean group differences were calculated by subtracting the mean relative source locations in the control group from those in the dyslexic group.
} 
dyslexic and normally literate children in medial-lateral [mean group difference $=-0.04 \mathrm{~cm}, F(1,17)=$ $0.02, P<0.895]$ and inferior-superior directions [mean group difference $=-0.42 \mathrm{~cm}, F(1,17)=0.7, P<0.410]$ again become obvious in Fig. 3B (right).

\subsection{Mismatch field}

In all subjects, standard and deviant wave forms in both the tone and speech blocks could be derived. Fig. 4 reveals the syllable data (standard and deviant wave forms) averaged for the dyslexics (left) as well as the controls (right). Both groups showed a significant difference between deviant and standard.

The three-way ANOVA of the RMS values did not indicate a group main effect implying that the mismatch responses of the dyslexics and controls did not differ in size. A statistically meaningful effect was yielded for the factor interval $[F(1,17)=5.6, P<0.030]$, i.e. the MMF activity was most prominent in the second evaluation window $\mathrm{B}$ (mean $\mathrm{RMS}$ value $=41$ vs. $46 \mathrm{fT}$ for interval A and B, respectively). The non-significant Group $\times$ Interval interaction emphasized the generality of the interval effect. The factor block (see Table 2) gained neither a significant influence by itself nor by an interaction with the variables group and/or interval, indicating that all subjects could automatically distinguish between simple and complex auditory stimuli.

\section{Discussion}

The present study examined two hypotheses: (1) dyslexia is characterized by a variant in the organisa- tion of the left-hemispheric auditory cortex, as marked by the sources of M80 and M210; and (2) the MMF to deviant consonant-vowel syllables is absent in dyslexic children. The experimental results confirmed the first assumption. The M210 generator was localized more anterior in the left temporal lobe of the dyslexic than of the control children. The magnitude of this effect was considerable with an average group difference of 1.47 $\mathrm{cm}$ for the absolute location of the M210 source. The effect was also clearly evident relative to the location of the M80 generator, although the latter did not systematically differ between the participant groups.

In adults, the middle-latency components, like P50 to tones, are directly associated with Heschl's gyrus [22], whereas the $\mathrm{N} 100 \mathrm{~m}$ arises from the depolarisation of pyramidal neurones in the planum temporale $[5,25]$. This view of generator locations is consistent with the present finding in the control children, with the middlelatent M80 being located anterior to the subsequently following M210. Probably in both the dyslexic and control children, primary auditory cortex was activated in the M80 latency range. In the subsequent processing stage, characterized by the M210, however, only the controls shifted activation to the posterior regions of the planum temporale. In contrast, in dyslexics, brain structures tended to be activated anterior to the M80 in Heschl's gyrus, i.e. in regions that are part of Wernicke's area but do not lie within the planum temporale.

One may suppose that, given no difference was found for the localisation of the M80 generator, the difference in M210 source cannot be attributed to gross anatomical variations. It deserves mentioning, however, that certain regions of the auditory cortex may have greater intersubject or developmental variability than other
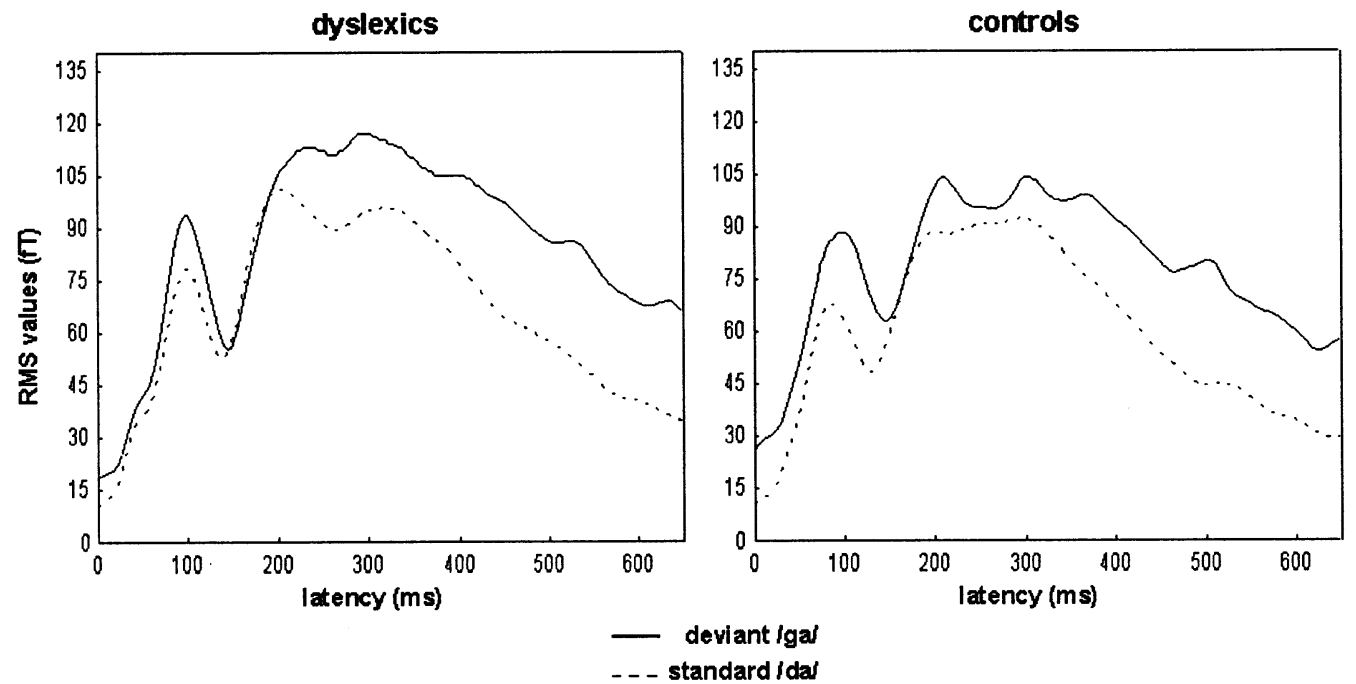

Fig. 4. Grand average of the RMS wave forms elicited by the syllables / da/ and /ga/ in the dyslexic and the control group. The dashed line characterizes the answer to the standard / da/, the solid line the response to the deviant $/ \mathrm{ga} /$. The pronounced difference between deviant and standard responses becomes evident within the latency range 280 to $380 \mathrm{~ms}$. 
regions. Assuming that the M210 but not the M80 is generated within a region with more natural variability, it is possible that the present group difference simply results from a difference in anatomy. Whether the origin of the atypical source configuration in children with dyslexia is structural or functional in nature cannot be settled at this point. Nevertheless, MEG technique provides a promising tool for revealing atypical organisation of the auditory cortex in children with dyslexia.

The second hypothesis that dyslexics reveal an absent MMF to deviant consonant-vowel syllables could not be confirmed by the present analyses. Both groups produced significant and normally pronounced MMFs. In both groups and across all stimulus conditions, the MMF was more prominent in the later time window B (280-380 ms), which is consistent with the rather long M210 latency.

The fact that an MMF group effect was not observed contrasts with the results reported by Kraus et al. [18] and also the more recently published data by SchulteKörne et al. [40], showing an absent/attenuated MMN to deviant syllables in learning disabled and dyslexic children. A factor possibly accounting for the different finding might be the characteristics of speech sounds. While Kraus' and Schulte-Körne's group presented synthetic syllables mainly differing in the frequency transitions of the second and third formant, we intended to improve the ecological validity of our study by using natural language. The spoken syllables /da/ and /ga/ diverged over and above that in the slowly changing parameters of the fundamental frequency providing information about the pitch of the speaker. As stated by Tallal et al. (e.g. Ref. [48]) dyslexic children do not show problems in slowly presented sounds but in the perception of short timed transient elements that may characterize certain stop consonants. Consequently, the normally pronounced $\mathrm{MMN}$ in our dyslexic sample might reflect variations in pitch rather than in the rapid changes of spectral characteristics in consonants. Further MEG studies utilizing carefully generated synthetic consonant-vowel syllables are needed in order to clarify the diverging mismatch results.

Finally, it should be kept in mind that Kraus et al. [18] and Schulte-Körne et al. [40] examined the electric $\mathrm{MMN}$, whereas we report findings for its magnetic counterpart. It may well be that the MMF reveals differences between the responses to standard and deviant stimuli more clearly that are blurred in the electrical recordings due to greater biological noise. Whenever the current sources in the cerebral cortex are predominantly tangential to the scalp, the MEG is likely to have a signal-to-noise ratio superior to that generated in an EEG, since the biological noise sums up to a radially oriented source $[5,6]$. The limited number of electrodes (9-19) used in the EEG studies could be another reason as to why a MMN has gone undetected in subjects with reading and spelling impairments: The different source configurations in dyslexic and control children, as found in the present study, might lead to a different scalp distribution of mismatch activity and consequently, an electrode array optimized for normal MMN topography may fail to detect deviant distributions. To resolve this issue, future studies should include either a high density electrode array or a combination of MEG and EEG.

In conclusion, our data suggest that dyslexics and normally literate children differ as to the organisation of their left-hemispheric auditory cortex. Whether this atypical configuration is specific to the auditory cortex of the left-hemisphere or is paralleled by a similar pattern in the right hemisphere should be examined in future studies monitoring the activation of both hemispheres.

\section{Acknowledgements}

The research presented here was supported by grants from the Deutsche Forschungsgemeinschaft. We wish to thank the children for their participation in the study as well as the parents for their co-operation. We gratefully acknowledge Professor Robert B. Freeman, Ph.D. for his helpful comments on a draft of this article.

\section{References}

[1] Brunswick N, McCrory E, Price CJ, Frith CD, Frith U. Explicit and implicit processing of words and pseudowords by adult developmental dyslexics. A search for Wernicke's Wortschatz? Brain 1999;122:1901-17.

[2] Dilling H, Mombour W, Schmidt MH. International Classification of Mental Diseases, ICD-10. Bern: Huber, 1991 German edition.

[3] Elberling C, Bak C, Kofoed B, Lebech J, Saermark K. Magnetic auditory responses from the human brain. Scandinavian Audiology 1980;9:185-90.

[4] Elberling C, Bak C, Kofoed B, Lebech J, Saermark K. Auditory magnetic fields from the human cortex: Influence of stimulus intensity. Scandinavian Audiology 1981;10:203-7.

[5] Elbert T. Neuromagnetism. In: Andrä W, Nowak H, editors. Magnetism in Medicine. New York: Wiley, 1998:190-262.

[6] Eulitz C, Eulitz H, Elbert T. Differential outcomes from magneto- and electroencephalography for the analysis of human cognition. Neuroscience Letters 1997;227:185-8.

[7] Farmer ME, Klein RM. The evidence for a temporal processing deficit linked to dyslexia: A review. Psychonomic Bulletin and Review 1995;2:460-93.

[8] Galaburda AM. Ordinary and extraordinary brain development: Anatomical variation in developmental dyslexia. Annals of Dyslexia 1987;39:67-80.

[9] Galaburda AM. Neuroanatomical basis of developmental dyslexia. Neurologic Clinics 1993;11:161-73.

[10] Galaburda AM, Livingstone M. Evidence for a magnocellular defect in developmental dyslexia. Annals New York Academy of Sciences USA 1993;682:70-82. 
[11] Galaburda AM, Sherman GF, Rosen GD, Aboitiz F, Geschwind N. Developmental dyslexia: Four consecutive patients with cortical anomalies. Annals of Neurology 1985;18:222-33.

[12] Galaburda AM, Corsiglia J, Rosen GD, Sherman GF. Planum temporale asymmetry, reappraisal since Geschwind and Levitsky. Neuropsychologia 1987;25:853-68.

[13] Grund M, Haug G, Naumann CL. Diagnostischer Rechtschreibtest für 4. Klassen, DRT 4. Weinheim: Beltz, 1994.

[14] Hari R. The neuromagnetic method in the study of the human auditory cortex. In: Grandori F, Hoke M, Romani GL, editors. Auditory Evoked Magnetic Fields and Electric Potentials, vol. 6. Basel: Karger, 1990:222-82.

[15] Humphreys P, Kaufmann WE, Galaburda AM. Developmental dyslexia in women: Neuropathological findings in three patients. Annals of Neurology 1990;28:727-38.

[16] Hynd GW, Semrud-Clikeman M, Lorys AR, Novey ES, Eliopulos D. Brain morphology in developmental dyslexia and attention deficit disorder/hyperactivity. Archives de Neurologie 1990;47:919-26.

[17] Kraus N, McGee T, Carrell T, Sharma A, Micco A, Nicol T. Speech-evoked cortical potentials in children. Journal of American Academy of Audiology 1993;4:238-48.

[18] Kraus N, McGee TJ, Carrell TD, Zecker SG, Nicol TG, Koch DB. Auditory neurophysiologic responses and discrimination deficits in children with learning problems. Science 1996;273:971-3.

[19] Kushch A, Gross-Glenn K, Jallad B, Lubs H, Rabin M, Feldman E, Duara R. Temporal lobe surface area measurements on MRI in normal and dyslexic readers. Neuropsychologia 1993;31:811-21.

[20] Larsen JP, Høien T, Lundberg I, Ødegaard H. MRI evaluation of the size and symmetry of the planum temporale in adolescents with developmental dyslexia. Brain and Language 1990;39:289301.

[21] Leonard CM, Voeller KKS, Lombardino LJ, Morris MK, Hynd GW, Alexander AW, Andersen HG, Garofalakis M, Honeyman JC, Mao J, Agee OF, Staab EV. Anomalous cerebral structure in dyslexia revealed with magnetic resonance imaging. Archives de Neurologie 1993;50:461-9.

[22] Liegois-Chauvel C, Musolino A, Badier J, Marquis P, Chauvel P. Evoked potentials recorded from the auditory cortex in man: Evaluation and topography of the middle-latency components. Electroencephalography and Clinical Neurophysiology 1994;92:204-14.

[23] Linder M, Grissemann H. Zürcher Lesetest, ZLT, 4th ed. Bern: Huber, 1980.

[24] Lubs HA, Smith S, Kimberling W, Pennington B, Gross-Glenn K, Duara R. Dyslexia subtypes: Genetics, behavior, and brain imaging. In: Plum F, editor. Research Publications, Association for Research in Nervous and Mental Disease, vol. 66. New York: Raven Press, 1988:139-47.

[25] Lütkenhöner B, Steinsträter O. High-precision neuromagnetic study of the functional organization of the human auditory cortex. Audiology Neurootology 1998;3:191-213.

[26] McGee T, Kraus N. Auditory development reflected by middle latency response. Ear and Hearing 1996;17:419-29.

[27] Meis R. Diagnostischer Rechtschreibtest 4-5, DRT 4-5. Weinheim: Beltz, 1970.

[28] Müller R. Diagnostischer Rechtschreibtest für 2. Klassen, DRT 2, 2nd ed. Weinheim: Beltz, 1983.

[29] Müller R. Diagnostischer Rechtschreibtest für 3. Klassen, DRT 3. Weinheim: Beltz, 1983.

[30] Näätänen R. Attention and Brain Function. Hillsdale, NJ: Erlbaum, 1992.

[31] Oades RD, Dittmann-Balcar A, Schepker R, Eggers C, Zerbin D. Auditory event-related potentials (ERPs) and mismatch negativity (MMN) in healthy children and those with attention-deficit or tourette/tic symptoms. Biological Psychology 1996;43:163-85.
[32] Oades RD, Dittmann-Balcar A, Zerbin D. Development and topography of auditory event-related potentials (ERPs): Mismatch and processing negativity in individuals $8-22$ years of age. Psychophysiology 1997;34:1-17.

[33] Paetau R, Ahonen A, Salonen O, Sams M. Auditory evoked magnetic fields to tones and pseudowords in healthy children and adults. Journal of Clinical Neurophysiology 1995;12:17785.

[34] Pantev C, Lütkenhöner B, Hoke M, Lehnertz K. Comparison between simultaneously recorded auditory-evoked magnetic fields and potentials elicited by ipsilateral, contralateral, and binaural tone burst stimulation. Audiology 1986;25:54-61.

[35] Pantev C, Bertrand O, Eulitz C, Verkindt C, Hampson S, Schuirer G, Elbert T. Specific tonotopic organizations of different areas of the human auditory cortex revealed by simultaneous magnetic and electric recordings. Electroencephalography and Clinical Neurophysiology 1995;94:26-40.

[36] Pennington BF. Genetics of learning disabilities. Journal of Child Neurology 1995;10(1):69-77.

[37] Rack JP. Dyslexia: The phonological deficit hypothesis. In: Fawcett A, Nicolson R, editors. Dyslexia in Children: Multidisciplinary Perspectives. Wheatsheaf: Harvester, 1994:5-37.

[38] Rathenow P, Laupenmühlen D, Vöge J. Westermann Rechtschreibtest $6+$, WRT $6+$, 2nd ed. Braunschweig: Westermann, 1981.

[39] Rumsey JM, Donohue BC, Brady DR, Nace K, Giedd JN, Andreason P. A magnetic resonance imaging study of planum temporale asymmetry in men with developmental dyslexia. Archives de Neurologie 1997;54:1481-9.

[40] Schulte-Körne G, Deimel W, Bartling J, Remschmidt H. Auditory processing and dyslexia: Evidence for a specific speech processing deficit. NeuroReport 1998;9:337-40.

[41] Schultz RT, Cho NK, Staib LH, Kier LE, Fletcher JM, Shaywitz SE, Shankweiler DP, Katz L, Gore JC, Duncan JS, Shaywitz BA. Brain morphology in normal and dyslexic children: The influence of sex and age. Annals of Neurology 1994;35:732-42.

[42] Shaywitz SE, Shaywitz BA, Fletcher JM, Escobar MD. Prevalence of reading disability in boys and girls: Results of the Connecticut Longitudinal Study. Journal of the American Medical Association 1990;264:998-1002.

[43] Shaywitz SE, Shaywitz BA, Pugh KR, Fulbright RK, Constable RT, Mencl WE, Shankweiler DP, Liberman AM, Skudlarski P, Fletcher JM, Katz L, Marchione KE, Lacadie C, Gatenby C, Gore JC. Functional disruption in the organization of the brain for reading in dyslexia. Proceedings of the National Academy of Science USA 1998;95:2636-41.

[44] Stein L, Kraus N, McGee T, Koch DB. New developments in the clinical application of auditory evoked potentials with children with multiple handicaps. Scandinavian Audiology 1995;24(41):18-30.

[45] Steinmetz H. Structure, function and cerebral asymmetry: In vivo morphometry of the planum temporale. Neuroscience and Biobehavioral Reviews 1996;20:587-91.

[46] Steinmetz H, Rademacher J, Jäncke L, Huang Y, Thron A, Zilles K. Total surface of temporoparietal intrasylvian cortex: Diverging left-right asymmetries. Brain and Language 1990;39:357-72.

[47] Steinschneider M, Kurtzberg D, Vaughan HG. Event-related potentials in developmental neuropsychology. In: Rapin I, Segalowitz SJ, editors. Handbook of Neuropsychology, vol. 6. Amsterdam: Elsevier, 1992:239-99.

[48] Tallal P, Miller S, Fitch RH. Neurobiological basis of speech: A case for the preeminence of temporal processing. Annals New York Academy of Sciences USA 1993;682:27-47.

[49] Tonnquist-Uhlén I. Topography of auditory evoked cortical potentials in children with severe language impairment. Scandinavian Audiology 1996;25(44):3-40. 
[50] Tonnquist-Uhlén I, Borg E, Spens KE. Topography of auditory evoked long-latency potentials in normal children, with particular reference to the N1 component. Electroencephalography and Clinical Neurophysiology 1995;95:34-41.

[51] von Economo C, Horn L. Über Windungsrelief, Masse und Rindenarchitektonik der Supratemporalfläche, ihre individuellen und ihre Seitenunterschiede. Zeitschrift für Neurologie und Psychiatrie 1930;130:678-757.

[52] Wagner RK, Torgesen JK. The nature of phonological processing and its causal role in the acquisition of reading skills. Psychological Bulletin 1987;101:192-212.
[53] Weiss R. Culture Fair Intelligence Test, Scale 2, CFT 20, 2nd ed. Göttingen: Hogrefe, 1987 German adaptation.

[54] Weiss R, Osterland J. Culture Fair Intelligence Test, Scale 1, CFT 1, 3rd ed. Braunschweig: Westermann, 1979 German adaptation.

[55] Welte V. Der Mottier-test, ein Prüfungsmittel für die Lautdifferenzierungsfähigkeit und die Auditive Merkfähigkeit. SpracheStimme-Gehör 1981;5:121-5.

[56] Yoshiura T, Ueno S, Iramina K, Masuda K. Human middle latency auditory evoked magnetic fields. Brain Topography 1996;8:291-6. 